\title{
Effect of Early Protective Lung Ventilation on Mortality and Hemodynamics in Patients With Acute Myocardial Infarction Complicated With Cardiogenic Shock and Pulmonary Edema Undergoing Emergency Interventional Therapy
}

Hao Yang

Tianjin Medical University

Chuyi Han

Tianjin Medical University

Xiaoling Guo

Tianjin Chest Hospital

Hongliang Wang

Tianjin Chest Hospital

Yanda Wu

Tianjin Medical University

Hongliang Cong ( $\sim$ hongliangcong@126.com )

Tianjin Chest Hospital

\section{Research Article}

Keywords: Early protective lung ventilation, Acute myocardial infarction, Cardiogenic shock, Pulmonary edema, Mortality

Posted Date: November 30th, 2021

DOI: https://doi.org/10.21203/rs.3.rs-1057961/v1

License: (c) (i) This work is licensed under a Creative Commons Attribution 4.0 International License.

Read Full License 


\section{Abstract}

Background: To investigate the effect of early protective lung ventilation (EPLV) on mortality and hemodynamic parameters in patients with acute myocardial infarction complicated with cardiogenic shock (CS) and pulmonary edema undergoing emergency percutaneous coronary intervention (PCI).

Methods: From January 2015 to June 2017, patients with acute myocardial infarction complicated with CS and pulmonary edema were admitted to the Tianjin chest Hospital. Based on the use of a mechanical ventilator, patients were divided into the EPLV and Non-invasive ventilation (NIV) groups. Hemodynamic indexes and in-hospital mortality of patients between the two groups was analyzed.

Results: The EPLV group consisted of 51 patients and the NIV group consisted of 38 patients. The difference in mortality rates was statistically significant between the EPLV and NIV groups $(P=0.01)$. Central venous pressure, pulmonary artery pressure, and pulmonary capillary wedge pressure after emergency $\mathrm{PCl}$ in the EPLV group were lower compared to patients in the NIV group $((P<0.05)$. Man arterial pressure in patients in the EPLV group was higher compared to NIV patients $(P<0.05)$. Logistic regression analysis showed that EPLV did not increase the risk of mortality $(P=0.37, \mathrm{OR}=2.16,95 \% \mathrm{Cl}$ $(0.31,9.52))$.

Conclusion: EPLV resulted in lower mortality and improved hemodynamic function in patients with acute myocardial infarction complicated with CS and pulmonary edema undergoing emergency PCl.

\section{Background}

$\mathrm{CS}$ is the leading cause of death in geriatric AMI patients. PCI significantly improves prognosis and reduces the incidence of ischemic complications in patients with $\mathrm{AMI}$, especially in high-risk patients. However, this advantage is based on patients receiving prompt emergency $\mathrm{PCl}(1,2)$. This brings challenges to the use of mechanical assisted ventilation (MV), as patients who need MV have lower left ventricular function, unstable hemodynamics, and higher Killip grading (3). Improvement in oxygenation using Intra-Aortic Balloon Counterpulsation (IABP) alone was not apparent in a previous study (4), while other mechanical aids such as left ventricular assist (Impella, ECMO) have not been widely used to treat patients due to inadequate treatment experience, complications, and high costs. Furthermore, the emergency use of $\mathrm{PCl}$ in AMI patients with cardiogenic shock have not been widely used for interventional heart disease. (5-7)

It is not unusual to administer non-invasive (NIV) or invasive mechanical ventilatory (IMV) support to patients with acute left heart failure induced by acute hypoxemic respiratory failure with concurrent $\mathrm{PCl}$ (8). NIV has been widely used to treat patients with CS and acute pulmonary edema. It has been shown to promote hemodynamic stability and increase survival (9). However, IMV has been shown to induce adverse effects on cardiac output and systemic perfusion in AMI patients. Furthermore, administration of IMV in CS patients is usually avoided (10). Several studies have suggested that positive pressure ventilation (PEEP) reduces cardiac output and organ perfusion and affects hemodynamic stability (11). 
However, these studies were based on patients with ARDS. Whether this is observed in AMI patients with CS needs to be investigated.

\section{Hypothesis}

Protective lung ventilation (EPLV) strategy consists of a small tidal volume with appropriate positive endexpiratory pressure (PEEP). Limited data is available to address the mode of respiratory support in the setting of acute MI. Hence, we investigated the effect of EPLV on mortality and hemodynamic parameters in patients with $\mathrm{AMI}$ combined with CS who were administered emergency $\mathrm{PCl}$.

\section{Methods}

\section{Patients}

This was a double-center retrospective study conducted from January 2015 to June 2019. Patients with acute myocardial infarction complicated with CS were admitted to the Tianjin chest Hospital were enrolled for the study. This study was performed in accordance with the relevant guidelines and regulations. This study was approved by the ethics committee of the Tianjin chest Hospital and conformed to all local and state ethical guidelines.

\section{Inclusion Criteria}

Patient inclusion criteria included the following (1) Patients older than18 years. (2) AMI patients with CS manifestations of acute pulmonary edema. Diagnostic criteria for AMI (3) included: an elevation of cardiac troponin levels with at least one measurement above the 99th percentile upper reference limit accompanied by at least one of the following: 1) ST-T changes in the electrocardiogram or newly developed complete left bundle branch conduction block, the appearance of Q wave.2) Persistent chest pain for more than 30 minutes. 3) Echocardiography showing local ventricular wall movement abnormalities.

Diagnostic criteria for cardiogenic shock (1) included 1) systolic blood pressure (SBP) $<90 \mathrm{mmHg}$ in the absence of low blood volume, or vasoactive drugs were required to maintain systolic blood pressure $\geq 90 \mathrm{mmHg}$; 2) Accompanied with tissue hypoperfusion: urine volume $\leq 20 \mathrm{ml} / \mathrm{h}$; Changes in central nervous system function; 3 ) Peripheral vasoconstriction: cold, damp skin and cyanosis at the extremities. Other causes such as hypotension due to pain, vagal reflex, arrhythmia, medication, or bleeding were excluded. Criteria for acute pulmonary edema were the presence of severe dyspnea, the presence of moist rales in both lungs, and a chest radiograph showing interstitial lung or alveolar infiltration.

Patients requiring complete emergency $\mathrm{PCl}$ treatment.All patients were immediately evaluated at the bedside using an 18 lead ECG after admission. Myocardial enzymes, troponin-I, Nt-pro BNP, blood routine, renal function, electrolyte, blood coagulation, bedside chest X-ray, and chocardiography were also evaluated. Patients were administered aspirin $300 \mathrm{mg}$, clopidogrel $300 \mathrm{mg}$ or ticagrelor $180 \mathrm{mg}$ via oral or gastric tube. Patients were also closely monitored for heart rate, blood pressure, and blood oxygen levels. 
Venous access was established and patients were infused with diuretics, vasoactive drugs, and other active corrections for shock and heart failure. Patients with oxygen saturation $<90 \%$ before treatment were administered a non-invasive ventilator (NIV) or EPLV. Patients were divided into the EPLV and NIV group based on whether invasive ventilation was used.

NIV consisted of continuous positive airway pressure (CPAP) and bi-level positive airway pressure (BiPAP) and was delivered through face masks. Expiratory positive airway pressure (EPAP), inspiratory positive airway pressure (IPAP), and FiO2 were then monitored.

EPLV group had acute pulmonary edema respiratory failure, arterial blood gas analysis $\mathrm{PO}_{2}<50 \mathrm{mmHg}$, $\mathrm{PH}<7.20$, respiratory frequency $>40$ times/min, and continual blood oxygen saturation of less than $80 \%$. Diuretics, vasoactive drugs, common mask, and/or nasal catheter oxygen inhalation methods are unable to alleviate symptoms while non-invasive ventilators are unable to rapidly correct refractory hypoxemia (4). The EPLV treatment procedure consisted of patients being administered anesthesia followed by bedside endotracheal intubation and ventilator-assisted breathing. Medium level $\left(4-10 \mathrm{~cm} \mathrm{H}_{2} \mathrm{O}\right)$ with positive end expiratory pressure ventilation (PEEP) mode was used. Respiratory parameters were adjusted based on blood oxygen saturation and blood gas analysis (oxygen partial pressure, carbon dioxide partial pressure, $\mathrm{PH}$ value) to maintain blood oxygen saturation levels $>90 \%$.

IABP: Patients with CS underwent left or right femoral artery puncture in the emergency room, and the IABP catheter was placed bedside. When oxygen saturation levels were greater than $90 \%$, patients were transferred to the catheter room through the green channel.

$\mathrm{PCl}$ :Emergency coronary angiography and interventional therapy were performed based on published guidelines (3). TIMI grading was used to determine the blood flow in the infarct-related arteries. Significant stenosis was defined when lumen stenosis of the anterior descending branch, circumflex branch, right coronary artery, or major branch was greater than $75 \%$, and that of the left main trunk was greater than $50 \%$. After angiography was performed to determine vascular lesions, percutaneous coronary intervention ( $\mathrm{PCl}$ ) was performed based on the characteristics of the lesions. Treatment strategies for each patient were decided by two experienced cardiologists.

5) Hemodynamics monitoring

Right internal jugular vein puncture was performed immediately after surgery under local anesthesia, and a Swan-Ganz balloon floating catheter was inserted for continuous hemodynamic monitoring. Central venous pressure (CVP), pulmonary artery pressure (PAP), pulmonary capillary wedge pressure (PCWP) and mean arterial pressure (MAP) were recorded immediately and then subsequently after 3 hours, 6 hours, 12 hours, 24 hours, and 48 hours after surgery.

\section{Statistical Statistics}

SPSS 18.0 (SPSS, Inc., Chicago, IL, USA) software was used for statistical analyses. Continuous variables were expressed as mean with standard deviation (SD). Comparisons between continuous variables were 
performed using t-test or analysis of variance. Categorical variables were expressed as frequency and percentage. Repeated analyses of variances demonstrated differences in hemodynamic index changes at each time point. T-test was used for comparison between the two groups. Comparisons between categorical variables were compared using the chi-square test or Fisher's exact test. Statistically significant variables identified in univariate analysis (variable of $P<0.05$ ) were used for multivariable logistic regression analysis. Multiple logistic regression analysis identified risk factors of in-hospital mortality. A p-value of $\leq 0.05$ was considered statistically significant.

\section{Results}

\section{Baseline characteristics of the selected patients}

1693 patients who met the inclusion criteria were enrolled in this clinical observational study. The frequency of CS was $6.32 \%(107 / 1693)$ and those complicated with acute left heart failure were $83.18 \%$ (89/107). 57.53\% (51/89) of the patients received EPLV while $42.69 \%(38 / 89)$ patients received NIV.

Table 1 shows the general and baseline characteristics of patients who received EPLV and NIV. There were no significant statistical differences between the two groups for FMC time, CK-MB, NT-pro BNP, etc. $(P>0.05)$.

Hemodynamic parameters in the postoperative period in the two groups are shown in Figure 1. CVP, PAP, PCWP, and MAP at $0 \mathrm{~h}, 3 \mathrm{~h}, 6 \mathrm{~h}, 12 \mathrm{~h}, 24 \mathrm{~h}, 48 \mathrm{~h}$, and $72 \mathrm{~h}$ after PCl was compared. Repeated analyses of variances showed differences in the hemodynamic index at each time point. There were no statistically significant differences in CVP, PAP, PCWP, and MAP between the two groups immediately after operation $(P>0.05)$. However, CVP, PAP, and PCWP values of EPLV patients decreased 3 hours after surgery, while MAP increased gradually. The differences were statistically significant $(P<0.05)$.

Mortality between the two groups is shown. For all patients with MV, patient mortality in the EPLV group was lower compared to the NIV group $(29.4 \%(15 / 51)$ vs. $55.3 \%(21 / 38))$. There were significant statistical differences between the two groups $\left(X^{2}=6.04, P=0.01\right)$.

Clinical characteristics of patients who survived versus those who died in the two groups are shown in Table 2. There were no significant statistical differences between the two groups for gender,smoking history, hypertension, WBC, HGB, PLT, Hs - CRP, LDL-C, etc. $(P>0.05)$. There were significant statistical differences between the two groups for age and diabetes $(P<0.05)$. ALT, CRE, blood glucose levels on admission, and NT- pro BNP levels were higher in patients who died compared to patients who survived. FMC time was longer in patients who died versus patients who survived. Postoperative TIMI blood flow $<3$ was observed more frequently in patients who died versus patients who survived.

Analysis of relevant risk factors for in-hospital mortality of patients with AMI complicated with CS with acute pulmonary edema is shown in Table 3. After adjusting for age, complicated disease, and other risk factors, multivariate binary logistic regression analyses showed that extended FMC time, kidney failure, 
and postoperative TIMI blood flow < grade 3 were independent risk factors for CS death after AMI $(P<0.05)$, while EPLV did not increase the risk of patient death.

\section{Discussion}

Our study demonstrated EPLV increased PCI success rate, reduced mortality rates,alleviated pulmonary edema, and restored proper hemodynamics in patients with AMI combined with CS.

CS is a serious complication observed in AMI patients with an average incidence of about $5 \%$ 15\%, with $1 / 3$ of patients manifesting CS at the time of admission

(12). Our study showed that the incidence of CS after AMI was $6.32 \%$, which was similar to previously published reports. The pathophysiological characteristics of AMI combined with CS are hypoxia of myocardial tissue and the decrease in cardiac output due to insufficiency of coronary artery volume. Regarding hemodynamics, PCWP is usually higher than $15 \mathrm{~mm} \mathrm{Hg}$, which leads to increased left ventricular filling pressure. Hence, CS patients are often associated with severe pulmonary edema $(5,13)$. Our study found that $83.18 \%$ of CS patients had severe pulmonary edema. Patients suffering from pulmonary edema have difficulty in undergoing complete emergency $\mathrm{PCl}$ (14). To correct hypoxia quickly, ventilator support is often required. We hypothesized that EPLV could alleviate pulmonary edema rapidly and achieve clinical hemodynamic benefits. We found that early application of IMV with moderate PEEP could promote hemodynamic stability. PEEP reduced left ventricular afterloading by reducing intrathoracic pressure and left ventricular preload by reducing the volume of venous return. This in turn relieved cardiac congestion, reduced the workload and metabolism of the respiratory muscles, reversed pulmonary vasoconstriction induced by hypoxia, and increased myocardial oxygen availability. These hemodynamic changes had a beneficial effect on patients with left ventricular dysfunction, who were sensitive to changes in afterloading.

Several studies on ARDS patients have suggested that mechanical ventilation was associated with an increased risk of death $(15,16)$. However, left ventricular function which depends on the influence of preload is normal in ARDS patients with sepsis $(16,17)$. Our study showed the mortality was significantly decreased in patients in the EPLV group. The decrease in cardiac output caused by PEEP was mainly due to a decrease in the volume of venous blood returning to the heart, resulting in a reduction in the preload (18). Therefore, EPLV in patients with CS had beneficial effects on hemodynamics as well as clinical endpoint events.

Numerous studies have demonstrated that active revascularization could significantly improve the prognosis of patients with AMI and CS (19). However, due to the patient's clinical status, excessive risk of interventional surgery, interventional techniques, and other factors, patients with cardiac shock often do not receive the required treatment $(14,20)$. The state of cardiopulmonary instability before surgery understandably delays the time of reperfusion therapy. Shortening treatment time could significantly benefit AHF patients. Rapid and active treatment is the key to prevent the deterioration of AHF. Our study found that the D to B time in the EPLV group was significantly lower compared to the NIV group. This 
suggested that EPLV could stabilize clinical symptoms and increase compliance in patients. EPLV therapy was found to facilitate gas exchange, replenish alveolar units, transfer water from alveoli and interstitial to the cycle, counteract pulmonary edema induced by hydrostatic forces, maintain airway, reverse acute hypoxic respiratory failure, improve breathing difficulties and respiratory distress $(10,21)$.

Our study demonstrated that increased FMC time, liver and renal failure, and postoperative TIMI blood flow $<3$ grade were independent risk factors for death in CS. EPLV was found to not increase the risk of death. This suggested that patients with CS could benefit from EPLV during emergency PCI.

In summary, the prognosis of AMI patients relied heavily on the implementation of early effective reperfusion of ischemic myocardium. However, because of the presence of heart failure and pulmonary edema, mechanical assisted ventilation is essential. For patients with AMI combined with CS, EPLV increased the safety, reliability, and success rate of surgery, and reduced the mortality rates of patients with AMI combined with CS. Furthermore, it shortened hospitalization times.

\section{Study Limitations}

This was a retrospective study with a limited number of patients. In addition, long-term follow-ups were not performed. For patients undergoing emergency interventional therapy, we only used IABP as mechanical support. Hence, the impact of more advanced mechanical-assisted support treatments such as ECMO and LVADs on acute AMI patients was not studied. Our findings suggest EPLV could be safely used in patients who present with severe hemodynamic impairment, such as acute pulmonary edema or respiratory failure and refractory hypoxemia, or for patients who were treated with vasoactive agents and diuretics. From our experience, EPLV could be possible during angiography and PCI. The survival rate of patients could be improved using EPLV together with early emergency interventional therapy.

All authors declare no conflicts of interest.

\section{Conclusion}

EPLV was not only able to decrease mortality but also improve hemodynamics in patients with acute myocardial infarction complicated with cardiogenic shock and pulmonary edema undergoing emergency $\mathrm{PCl}$.

\section{Abbreviations}

MV: mechanical assisted ventilation

IABP: Intra-Aortic Balloon Counterpulsation

EPLV: effect of early protective lung ventilation

CS: cardiogenic shock 
PCl: percutaneous coronary intervention

NIV: Non-invasive ventilation

IMV: invasive mechanical ventilatory

PEEP: positive pressure ventilation

SBP: systolic blood pressure

CPAP: continuous positive airway pressure

BiPAP: bi-level positive airway pressure

EPAP: Expiratory positive airway pressure

IPAP: inspiratory positive airway pressure

CVP:Central venous pressure

PAP: pulmonary artery pressure

PCWP: pulmonary capillary wedge pressure

MAP: mean arterial pressure

SD: standard deviation

\section{Declarations}

\section{Funding}

The study is supported by Special Projects on Basic Research Cooperation of Beijing, Tianjin and Hebei (19JCZDJC65600Z).

\section{Competing interests}

All authors declare that there is no conflict interest.

\section{Ethics approval and consent to participate}

This study was approved by the ethics committee of Tianjin chest Hospital and conformed to all local and state ethical guidelines. All participants in the study have signed informed consent. All authors listed have read the complete manuscript and have approved submission of the paper. The manuscript is truthful original work without fabrication, fraud or plagiarism. All authors declare that there is no conflict interest. 


\section{Consent for publication}

Not applicable.

\section{Acknowledgements}

None.

\section{Availability of data and materials}

The datasets used and/or analysed during the current study are available from the corresponding author on reasonable request.

\section{Authors' contributions}

$\mathrm{HY}$ take responsibility for the authenticity of this work and take responsibility for the integrity of the data and the accuracy of the data analysis. The study design was performed by $\mathrm{HL} C$, and $\mathrm{H} Y$; Literature search was performed by $\mathrm{H} Y$, and $\mathrm{CY} H$; Clinical studies and data acquisition was performed by $\mathrm{XL} G$, YD W and HL W; Statistical analysis was performed by $\mathrm{H} \mathrm{Y}$; Manuscript writing was performed by $\mathrm{H} Y$ and Manuscript revision/review were performed by $\mathrm{HL}$ C. All authors read and approved the final manuscript.

\section{References}

1. Tehrani BN, Basir MB, Kapur NK. Acute myocardial infarction and cardiogenic shock: Should we unload the ventricle before percutaneous coronary intervention? Prog Cardiovasc Dis. 2020;63:60722.

2. Vahdatpour C, Collins D, Goldberg S. Cardiogenic Shock. J Am Heart Assoc. 2019;8:e011991.

3. Ibanez B, James S, Agewall S, Antunes MJ, Bucciarelli-Ducci C, Bueno H, et al. 2017 ESC Guidelines for the management of acute myocardial infarction in patients presenting with ST-segment elevation: The Task Force for the management of acute myocardial infarction in patients presenting with STsegment elevation of the European Society of Cardiology (ESC). Eur Heart J. 2018;39:119-77.

4. Liu H, Wu X, Zhao X, Zhu P, Han L. Intra-aortic balloon pump combined with mechanical ventilation for treating patients aged $>60$ years in cardiogenic shock: Retrospective analysis. J Int Med Res. 2016;44:433-43.

5. Lorente V, Aboal J, Garcia C, Sans-Roselló J, Sambola A, Andrea R, et al. Anemia in patients with high-risk acute coronary syndromes admitted to Intensive Cardiac Care Units. J Geriatr Cardiol. 2020;17:35-42.

6. Levy B, Bastien O, Karim B, Cariou A, Chouihed T, Combes A, et al. Experts' recommendations for the management of adult patients with cardiogenic shock. Ann Intensive Care. 2015;5:52.

7. Shah AH, Puri R, Kalra A. Management of cardiogenic shock complicating acute myocardial infarction: A review. Clin Cardiol. 2019;42:484-93. 
8. van Diepen S, Hochman JS, Stebbins A, Alviar CL, Alexander JH, Lopes RD. Association Between Delays in Mechanical Ventilation Initiation and Mortality in Patients With Refractory Cardiogenic Shock. JAMA Cardiol. 2020;5:965-7.

9. Wiesen J, Ornstein M, Tonelli AR, Menon V, Ashton RW. State of the evidence: mechanical ventilation with PEEP in patients with cardiogenic shock. Heart. 2013;99:1812-7.

10. Mebazaa A, Tolppanen H, Mueller C, Lassus J, DiSomma S, Baksyte G, et al. Acute heart failure and cardiogenic shock: a multidisciplinary practical guidance. Intensive Care Med. 2016;42:147-63.

11. Moghaddam N, van Diepen S, So D, Lawler PR, Fordyce CB. Cardiogenic shock teams and centres: a contemporary review of multidisciplinary care for cardiogenic shock. ESC Heart Fail. 2021;8:988-98.

12. Chioncel O, Parissis J, Mebazaa A, Thiele H, Desch S, Bauersachs J, et al. Epidemiology, pathophysiology and contemporary management of cardiogenic shock - a position statement from the Heart Failure Association of the European Society of Cardiology. Eur J Heart Fail. 2020;22:131541.

13. Pesaro AE, Katz M, Katz JN, Barbas CS, Makdisse MR, Correa AG, et al. Mechanical Ventilation and Clinical Outcomes in Patients with Acute Myocardial Infarction: A Retrospective Observational Study. PLoS One. 2016;11:e0151302.

14. Masip J, Peacock WF, Price S, Cullen L, Martin-Sanchez FJ, Seferovic P, et al. Indications and practical approach to non-invasive ventilation in acute heart failure. Eur Heart J. 2018;39:17-25.

15. Ketcham SW, Sedhai YR, Miller HC, Bolig TC, Ludwig A, Co I, et al. Causes and characteristics of death in patients with acute hypoxemic respiratory failure and acute respiratory distress syndrome: a retrospective cohort study. Crit Care. 2020;24:391.

16. Vieillard-Baron A, Matthay M, Teboul JL, Bein T, Schultz M, Magder S, et al. Experts' opinion on management of hemodynamics in ARDS patients: focus on the effects of mechanical ventilation. Intensive Care Med. 2016;42:739-49.

17. Lopez Saubidet I, Maskin LP, Rodríguez PO, Bonelli I, Setten M, Valentini R. Mortality in patients with respiratory distress syndrome. Med Intensiva. 2016;40:356-63.

18. Vallabhajosyula S, Dunlay SM, Murphree DH, Jr., Barsness GW, Sandhu GS, Lerman A, et al. Cardiogenic Shock in Takotsubo Cardiomyopathy Versus Acute Myocardial Infarction: An 8-Year National Perspective on Clinical Characteristics, Management, and Outcomes. JACC Heart Fail. 2019;7:469-76.

19. Longo S, Siri J, Acosta C, Palencia A, Echegaray A, Chiotti I, et al. Lung recruitment improves right ventricular performance after cardiopulmonary bypass: A randomised controlled trial. Eur $\mathrm{J}$ Anaesthesiol. 2017;34:66-74.

20. Świątkiewicz I, Magielski P, Kubica J, Zadourian A, DeMaria AN, Taub PR. Enhanced Inflammation is a Marker for Risk of Post-Infarct Ventricular Dysfunction and Heart Failure. Int J Mol Sci. 2020;21.

21. Kim JH, Sunkara A, Varnado S. Management of Cardiogenic Shock in a Cardiac Intensive Care Unit. Methodist Debakey Cardiovasc J. 2020;16:36-42. 


\section{Tables}

Table1. aseline data between patients who received EPLV and NIV. 
Variable

\begin{tabular}{|c|c|c|c|}
\hline Age (year) & $68.55 \pm 7.27$ & $66.42 \pm 5.55$ & 0.14 \\
\hline Male $(n, \%)$ & $31(60.78 \%)$ & $24(63.16 \%)$ & 0.82 \\
\hline Smoking (n, \%) & $21(41.18)$ & $11(28.95)$ & 0.17 \\
\hline $\begin{array}{l}\text { Previous history of myocardial infarction ( } \mathrm{n} \text {, } \\
\% \text { ) }\end{array}$ & $9(17.65)$ & $5(13.16)$ & 0.39 \\
\hline Diabetes (n, \%) & $21(41.18)$ & $13(34.21)$ & 0.33 \\
\hline hypertension(n, \%) & $25(49.02)$ & 18(47.37) & 0.52 \\
\hline WBC $\left(\times 10^{9 / L}\right)$ & $12.79 \pm 3.40$ & $11.93 \pm 3.22$ & 0.23 \\
\hline HGB $(g / L)$ & $133.69 \pm 16.30$ & $137.14 \pm 23.75$ & 0.42 \\
\hline PLT (g/L) & $214.50 \pm 60.16$ & $224.08 \pm 65.87$ & 0.48 \\
\hline $\mathrm{Hs}-\mathrm{CRP}(\mathrm{mg} / \mathrm{dl})$ & $115.59 \pm 65.46$ & $111.54 \pm 41.67$ & 0.74 \\
\hline CRE (umol/L) & $117.51 \pm 48.46$ & $113.96 \pm 50.86$ & 0.74 \\
\hline $\operatorname{ALT}(\mathrm{U} / \mathrm{L})$ & $162.05 \pm 76.62$ & $138.44 \pm 60.41$ & 0.12 \\
\hline LDL-C (mmol/l) & $3.35 \pm 0.98$ & $3.05 \pm 0.92$ & 0.13 \\
\hline $\mathrm{CK}-\mathrm{MB}(\mathrm{ng} / \mathrm{ml})$ & $297.67 \pm 101.49$ & $266.78 \pm 81.19$ & 0.13 \\
\hline $\mathrm{TNI}(\mathrm{ng} / \mathrm{ml})$ & $7.68 \pm 1.99$ & $7.52 \pm 1.60$ & 0.7 \\
\hline NT- pro BNP (pg/ml) & $5908.68 \pm 5327.03$ & $\begin{array}{l}7876.03 \\
\pm 4373.41\end{array}$ & 0.07 \\
\hline Admission blood glucose (mmol/L) & $9.76 \pm 3.10$ & $10.72 \pm 3.19$ & 0.12 \\
\hline FMC time $(\mathrm{h})$ & $14.06 \pm 4.16$ & $13.87 \pm 4.05$ & 0.82 \\
\hline TIMI blood flow 0-1 (n, \%) & $28(54.90)$ & $20(52.63)$ & 0.83 \\
\hline Anterior wall myocardial infarction & $21(41.18)$ & $17(44.74)$ & 0.4 \\
\hline Vascular localization of criminals $(n, \%)$ & & & 0.14 \\
\hline LM & $17(33.33)$ & $6(15.79)$ & \\
\hline LAD & $19(37.26)$ & $15(39.47)$ & \\
\hline LCX & $11(21.57)$ & $9(23.69)$ & \\
\hline RCA & $4(7.84)$ & $8(21.05)$ & \\
\hline Number of diseased vessels $(n, \%)$ & & & 0.59 \\
\hline Single branch & $\begin{array}{l}6(5.88) \\
\text { e } 12 / 16\end{array}$ & $4(10.53)$ & \\
\hline
\end{tabular}


WBC: white blood count; HGB: hemoglobin; PLT: blood platelet; LDL-C: low-density lipoprotein cholesterol; Hs - CRP: high sensitive $C$ reactive protein; CRE: serum creatinine; ALT: Alanine aminotransferase; CK MB:creatine kinase B(CK-MB); TNI: cardiac troponin T; NT- pro BNP: N-terminal pro-brain natriuretic peptide; FMC time: First medical contact time; LM: Left Main; LAD: Left anterior descending artery; LCX: left circumflex artery; RCA: right coronary artery.

Table 2. Clinical Characteristics of patients who survived versus deceased. 


\begin{tabular}{|c|c|c|c|}
\hline Characteristics & Alive $(n=53)$ & Deceased $(n=36)$ & $P$ \\
\hline Age(year) & $69.1 \pm 6.66$ & $65.42 \pm 6.04$ & 0.01 \\
\hline Male $(n, \%)$ & $30(56.60)$ & $25(69.44)$ & 0.22 \\
\hline Smoking (n, \%) & $22(41.51)$ & $11(30.56)$ & 0.06 \\
\hline Diabetes (n, \%) & $15(28.30)$ & $19(52.78)$ & 0.02 \\
\hline Hypertension (n, \%) & $27(50.94)$ & $16(44.44)$ & 0.55 \\
\hline Previous history of myocardial infarction (n, \%) & $4(7.55)$ & $10(27.78)$ & 0.01 \\
\hline $\operatorname{ALT}(\mathrm{U} / \mathrm{L})$ & $169.68 \pm 73.56$ & $125.9 \pm 58.11$ & 0 \\
\hline CRE (umol/L) & $89.29 \pm 22.59$ & $155.31 \pm 51.66$ & 0 \\
\hline WBC $\left(\times 10^{9 / L}\right)$ & $12.55 \pm 3.36$ & $12.21 \pm 3.32$ & 0.64 \\
\hline $\mathrm{HGB}(\mathrm{g} / \mathrm{L})$ & $133.745 \pm 16.26$ & $137.68 \pm 24.07$ & 0.33 \\
\hline PLT (g/L) & $210.33 \pm 63.35$ & $230.75 \pm 59.97$ & 0.13 \\
\hline $\mathrm{Hs}-\mathrm{CRP}(\mathrm{mg} / \mathrm{dl})$ & $118.49 \pm 60.01$ & $107.06 \pm 50.36$ & 0.35 \\
\hline LDL-C (mmol/l) & $3.29 \pm 0.95$ & $3.12 \pm 0.98$ & 0.43 \\
\hline $\mathrm{TNI}(\mathrm{ng} / \mathrm{ml})$ & $7.38 \pm 1.62$ & $7.95 \pm 2.07$ & 0.15 \\
\hline NT- pro BNP (pg/ml) & $5800.08 \pm 4200.72$ & $8145.21 \pm 5794.91$ & 0.03 \\
\hline Blood glucose on admission $(\mathrm{mmol} / \mathrm{L})(\mathrm{mmol} / \mathrm{L})$ & $11.66 \pm 3.14$ & $11.66 \pm 3.14$ & 0 \\
\hline Anterior wall myocardial infarction & $23(43.40)$ & $15(41.67)$ & 0.23 \\
\hline FMC time (h) & $9.16 \pm 2.76$ & $11.69 \pm 3.14$ & 0.02 \\
\hline Postoperative TIMI blood flow (n, \%) & $21(39.62)$ & $27(75.00)$ & 0 \\
\hline $\operatorname{LM}(\mathrm{n}, \%)$ & $13(24.53)$ & $10(27.78)$ & 0.73 \\
\hline Three vessel lesions ( $n, \%)$ & $34(64.15)$ & $22(61.11)$ & 0.77 \\
\hline $\operatorname{MV}(\mathrm{n}, \%)$ & & & 0.01 \\
\hline EPLV & $36(67.92)$ & $15(41.67)$ & \\
\hline NIV & $17(32.08)$ & $21(55.26)$ & \\
\hline
\end{tabular}

Table 3. Analysis of risk factors related to hospital death after AMI complicated with cardiogenic shock. 


\begin{tabular}{llll} 
Variable & $\mathrm{P}$ & $\mathrm{OR}$ & $95 \% \mathrm{Cl}$ \\
\hline age & 0.09 & 0.34 & $0.10,1.17$ \\
\hline diabetes & 0.1 & 2.61 & $0.84,8.12$ \\
\hline Previous history of myocardial infarction & 0.8 & 0.79 & $0.14,4.68$ \\
\hline Liver insufficiency & 0.09 & 0.35 & $0.10,1.17$ \\
\hline Renal failure & 0.02 & 3.7 & $1.21,11.28$ \\
\hline NT- pro BNP & 0.96 & 0.97 & $0.32,2.97$ \\
\hline Anterior wall myocardial infarction & 0.3 & 1.82 & $0.59,5.62$ \\
\hline Admission blood glucose (mmol/L) & 0.06 & 0.34 & $0.11,1.02$ \\
\hline FMC time & 0.02 & 3.99 & $1.28,12.41$ \\
\hline Postoperative TIMI blood flow & 0.01 & 4.98 & $1.62,15.36$ \\
\hline Three vessel lesions & 0.38 & 0.61 & $0.20,1.83$ \\
\hline EPLV & 0.33 & 2.06 & $0.48,8.83$
\end{tabular}

Figures 


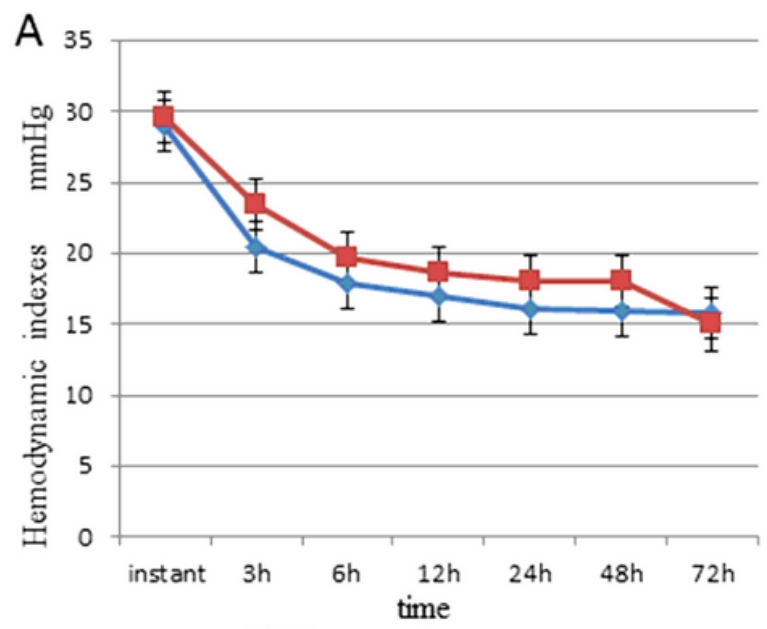

C

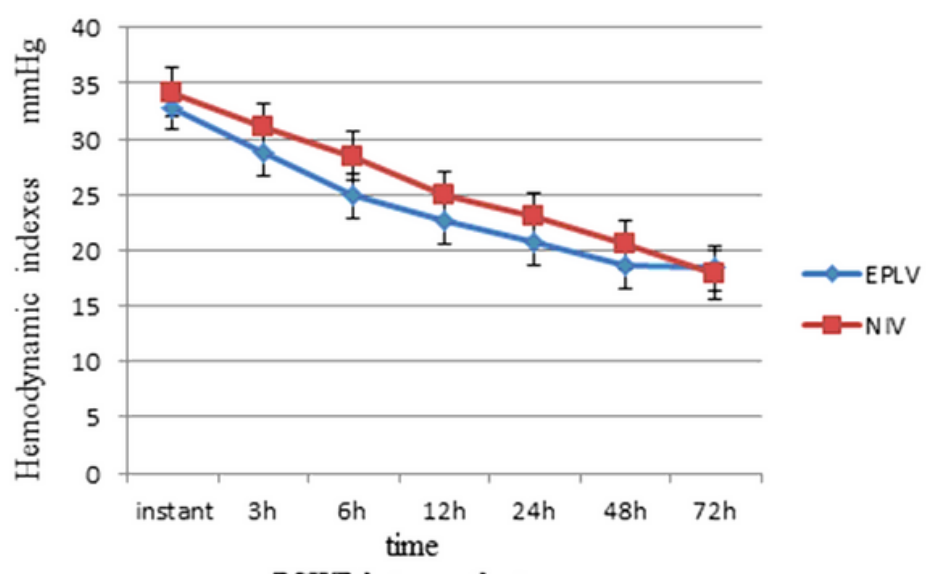

PCWP between the two group
B

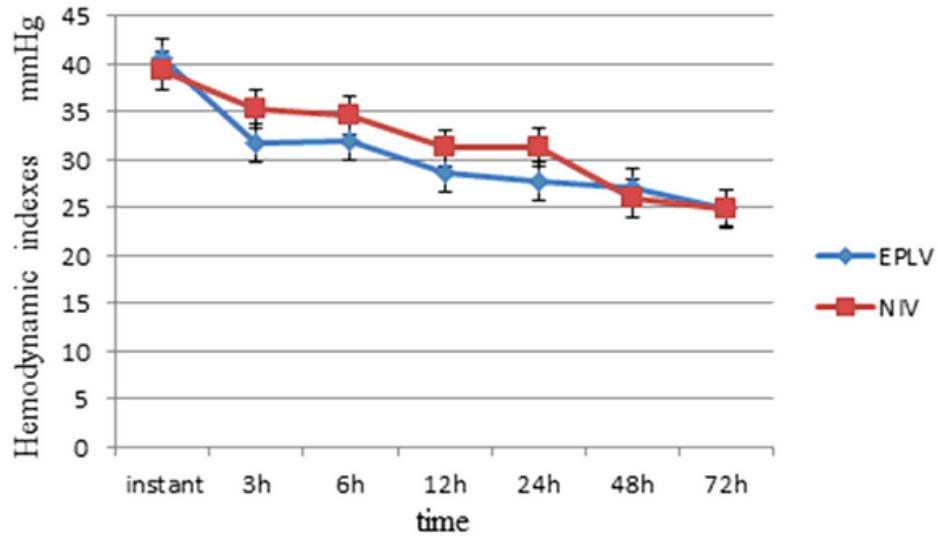

D

PAP between the two group

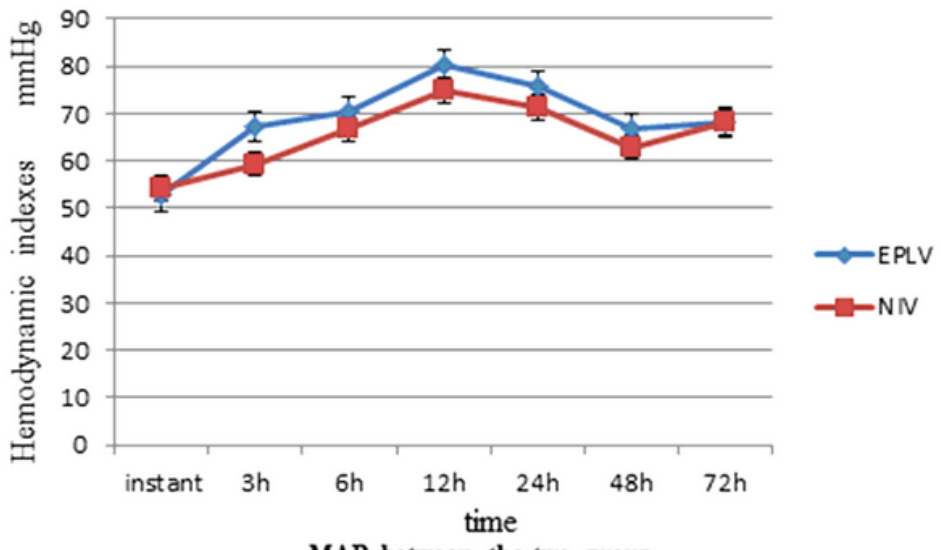

MAP between the two group

\section{Figure 1}

Changes in hemodynamic parameters after PCI in the EPLV and NIV groups 